\title{
Restoration of insulin receptor improves diabetic phenotype in T2DM mice
}

\author{
Yichen Wang, Heather Zhou, Oksana Palyha, and James Mu \\ Discovery, Preclinical and Early Development, MRL, Merck and Co., Inc., Kenilworth, New Jersey, USA.
}

Type 2 diabetes mellitus (T2DM), also known as adult-onset diabetes, is characterized by ineffective insulin action due to insulin resistance in key metabolic tissues. Insulin receptor (IR) plays an important role in insulin signal transduction, defect of which has been considered the fundamental cause of T2DM. IR content reduction in diabetes is one key contributor to the defective insulin signaling and diabetes progression. Rescuing IR levels by transgenic complementation has not been considered as a treatment option because it is limited by uncontrollable expression level, tissue selectivity, or developmental defects. In the current study, we demonstrated that single-dose adeno-associated virus (AAV) vector delivered expression of human IR (hIR) in the liver of inducible IR-knockout mice and significantly improved the diabetic phenotype caused by IR deletion during adulthood. Such an approach was also applied, for the first time to our knowledge, to treating ob/ ob mice, a model of severe T2DM attributed to superfluous calorie intake and insulin resistance. Interestingly, similar treatment with AAV-hIR had no obvious effect in healthy animals, indicative of low hypoglycemic risk as a consequence of potential excessive insulin action. The results described here support restoration of IR expression as a safe and effective T2DM therapeutic with a long-lasting profile.

Conflict of interest: All authors are employees of Merck Sharp and Dohme Corp., a subsidiary of Merck and $\mathrm{Co}$., Inc.

Copyright: (c) 2019, American Society for Clinical Investigation.

Submitted: September 14, 2018

Accepted: June 27, 2019

Published: August 8, 2019.

Reference information: JCl Insight. 2019;4(15):e124945. https://doi. org/10.1172/jci.insight.124945.

\section{Introduction}

Insulin is a key anabolic hormone that controls energy metabolism in mammals. Through binding to the insulin receptor (IR), insulin regulates blood glucose levels by promoting glucose uptake to skeletal muscle and fat and inhibiting hepatic glucose production (1). Blunted activation of insulin signal leading to diabetes mellitus involves insulin resistance and/or impaired $\beta$ cell function (2). Diabetes manifests as chronically elevated blood glucose levels that can result in a series of potentially life-threatening complications over time, such as cardiomyopathy, chronic kidney diseases, and neuropathy (3). More than 400 million people worldwide are suffering from diabetes (4). Among them, $90 \%$ of the patients are diagnosed as having type 2 diabetes mellitus (T2DM), which is characterized as being adult onset and primarily caused by impairment of insulin signaling in insulin-sensitive tissues (5).

IR interacts with insulin at the cell surface and is the crucial mediator of insulin signal transduction, including the PI3K/Akt pathway regulating metabolism and RAS/MAPK pathway modulating growth (6). Genetic mutation of IR leads to severe metabolic diseases, and deficiencies in IR and postreceptor mediators have been closely linked with insulin resistance, especially abnormalities associated with obesity (7). In addition, reduction of IR cellular content and kinase activity has been frequently reported in human subjects and animal models with diabetes (8-10). In the past 2-3 decades, researchers have tried to increase IR level in diabetic animals using genetic approaches to reconstitute insulin action across tissues. However, the results were mixed partially because of challenges with achieving desirable expression in key metabolic tissues and complete glucose normalization or, in the case of transgene animal studies, complication because of concerns over developmental issues (11-14). In the current study, we applied adeno-associated virus-mediated (AAV-mediated) IR expression in adult mice to restore primarily hepatic IR protein level, which significantly improved diabetic phenotypes in T2DM models without detectable side effects. These results support the therapeutic potential of such a strategy for T2DM treatment.

\section{Results}

$A A V$-human IR injection rescued the diabetic phenotype in tamoxifen-treated inducible IR-knockout mice. Gene therapy using AAV has shown its promise in clinical applications because of its low pathogenicity in humans 
and long-term transgene expression (15). As one of the most explored gene therapy vehicles, AAV has been actively applied for chronic diseases caused by a single gene defect (15). However, its potential for treating multifactorial diseases, such as diabetes, is less explored. IR level replenishment by a transgenic approach achieved some beneficial effects, although in the meantime revealed some adverse issues in T2DM animal models (14). Here, we tested whether IR expression via AAV delivery can be an effective route to accomplish the desired outcome of treating T2DM. To attain an adequate efficiency of IR expression, we applied the AAV9 and a truncated chicken $\beta$-actin promoter with CMV enhancer (short CB promoter) to assist human IR (hIR) expression (AAV-hIR) (see more details in the Methods section; Supplemental Figure 1A; supplemental material available online with this article; https://doi.org/10.1172/jci.insight.124945DS1). To investigate the rescuing effect of AAV-induced IR expression in animals with diabetes caused by IR deletion, we first generated inducible IR-knockout (iIR-KO) mice by crossing $\mathrm{IR}^{\mathrm{fl} / \mathrm{fl}}$ mice with transgenic mice expressing CreER(T2) recombinase. Without tamoxifen (Tm) treatment, this strain of mice behaves similarly to healthy mice with regard to blood glucose levels. Tm treatment of iIR-KO mice 3 to 4 months of age resulted in T2DM characteristic of hyperglycemia (Figure 1A), hyperinsulinemia (Figure 1B), and impaired insulin tolerance (Figure 1, F and G) and glucose tolerance (Figure 1, H and I). We also applied automated capillary Western blot analysis to quantify IR protein levels, which provides a higher level of accuracy compared with the traditional methods (16). Western blot results showed that treatment with Tm dramatically downregulated IR protein levels in the insulin-sensitive tissues, including liver, skeletal muscle, and epididymal white adipose tissue (eWAT) (Figure 1E). As a comparison, Tm treatment led to mild yet significant IR protein reduction in the heart but caused no significant changes in the kidney or brain (Figure $1 \mathrm{E})$. Among all the tissues examined, the liver was the most severely affected and lost more than $90 \%$ of IR. In accordance with this nearly complete loss of IR in the liver, iIR-KO mice showed decreased liver weight (Figure 1C) and liver triglycerides (TGs) (Figure 1D).

To test whether restoration of IR can rescue the adult-onset diabetic phenotype, we applied the AAVhIR treatment to Tm- or corn oil vehicle-treated iIR-KO mice. Animals at 3 months of age were subjected to Tm administration at the same time receiving AAV-hIR or control AAV. As expected, Tm treatment significantly increased blood glucose levels (Supplemental Figure 2A) 2 weeks after treatment. However, such change was not detected when AAV-hIR virus was co-injected with Tm (Supplemental Figure 2A). At the end of the third month, blood glucose of Tm-treated iIR-KO mice reached a higher level than what was observed 2 weeks after the initial Tm treatment (Figure 2A; compare to Supplemental Figure 2A). Glucose elevation caused by Tm was mitigated by AAV-hIR injection (Figure 2A). In addition, impaired insulin and glucose sensitivity of Tm-treated iIR-KO mice was fully rescued to a level comparable to that of the vehicle-treated group (Figure 2, D, E, G, and H). In line with the improved glucose metabolism, the reduction of liver TGs in Tm-treated iIR-KO mice was normalized by AAV-hIR injection (Figure 2F). Similarly, decreased plasma TGs observed in the Tm control group showed a trend of recovery in mice receiving AAV-hIR (Figure 2I). Tm-treated iIR-KO mice experienced severe lipoatrophy because of loss of IR in adipose tissue and showed a mild increase of circulating free fatty acids (FFAs) compared with the healthy controls. Yet AAV-hIR was able to counter such minor increase and induce significant inhibition of lipolysis (Supplemental Figure 3). Protein quantification revealed that AAV-hIR injection led to a significant increase of IR expression only in the liver of Tm-treated iIR-KO mice (Figure 2B), not in skeletal muscle (Supplemental Figure 2B) or eWAT (Supplemental Figure 2C). The higher IR protein level in liver was associated with an elevation of insulin-stimulated hepatic Akt phosphorylation (Figure 2C), indicating AAV-mediated IR expression enhanced downstream signal transduction.

Injection of AAV-hIR in corn oil vehicle-treated iIR-KO mice (i.e., nondiabetic animals) did not cause increase of IR protein level in any of the insulin-sensitive tissues (Figure 2B and Supplemental Figure 2, $\mathrm{B}$ and C). Consistent with the stable IR protein levels, AAV-hIR administration did not significantly affect any of the metabolic parameters examined in the vehicle-treated iIR-KO mice (Figure 2). These results demonstrated that liver-tropic IR restoration by AAV-hIR injection was sufficient to rescue the diabetic phenotype of Tm-treated iIR-KO mice, which have IR deficiency in multiple tissues. This approach works only under pathologically diabetic conditions and has minimal impact in nondiabetic mice.

IR protein level reduction in insulin-sensitive tissues of diabetic animal models. IR restoration rescued the diabetic phenotype in Tm-treated iIR-KO mice and provided proof of concept about the potential therapeutic value of AAV-hIR in animals with impaired IR. To test whether significant IR protein level change exists in common T2DM animals, we collected tissues from ad lib-fed wild-type (WT) and $o b / o b$ mice at 4 months of age; the 

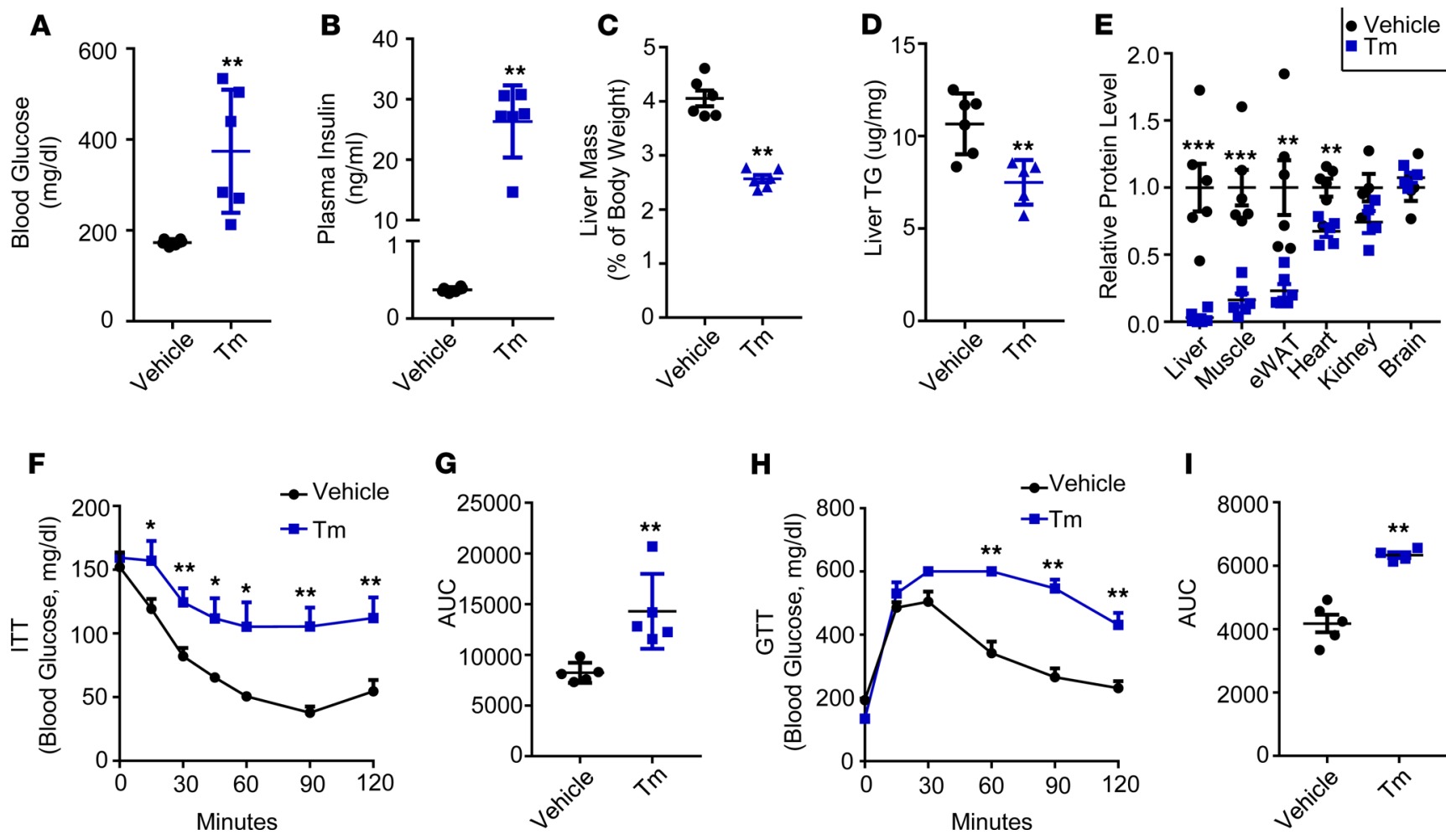

Figure 1. Tm treatment induced diabetic phenotype in ilR-KO mice. (A) Blood glucose and (B) plasma insulin levels were measured 2 weeks after Tm treatment $(n=6)$. (C) Liver weight and (D) liver TG levels were measured using terminal samples $(n=6)$. (E) IR protein levels were analyzed by Western blot in tissues as indicated in the panel. GAPDH was applied as an endogenous control in each tissue $(n=4-6)$. (F) Insulin tolerance and (H) glucose tolerance tests (ITT and GTT) are shown by representative glucose changes $(n=4-5)$. Glucose AUC ( $\mathbf{G}$ and $\mathbf{I})$ was calculated according to data in $\mathbf{F}$ and $\mathbf{H}$. Animals were sacrificed 7 weeks after Tm treatment and tissues were collected. Data shown as mean \pm SEM. Statistical analysis was conducted by Student's $t$ test $\left({ }^{*} P<0.05 ;{ }^{* *} P<0.01 ;{ }^{* *} P<0.001\right)$.

latter animals were insulin resistant and diabetic because of genetic leptin deficiency and obesity. Whole-tissue lysates were prepared from the liver, skeletal muscle, and eWAT of these animals. Meanwhile, membrane-bound proteins were extracted from the same samples (Figure 3A). IR protein levels were quantified by Western blotting using both the whole-tissue lysates and the membrane protein fractions. The results showed that, compared with that of WT mice, IR protein levels had a significant reduction in the whole-tissue lysate (Figure 3B; all reductions were greater than 40\%) and to a more dramatic degree in the membrane fraction (Figure 3C; reduction of $50 \%, 60 \%$, and $80 \%$ in liver, muscle, and eWAT, respectively) in all key metabolic tissues of $o b / o b$ mice. Studies in other models, such as diet-induced obese (DIO) mice, yielded similar results (Supplemental Figure 4). Collectively, these observations indicated that the reduction of IR level is a common phenomenon and may be the unifying pathophysiology underlying the defects of multiple T2DM models.

$A A V$-hIR injection rescued diabetic phenotype in ob/ob mice. To test whether restoring IR expression can alleviate the diabetic phenotype in $o b / o b$ mice, we injected AAV-hIR into $o b / o b$ and age-matched WT mice. Two weeks after AAV injection, the blood glucose levels of AAV-hIR-injected $o b / o b$ mice began to show significant reduction compared with that of control virus-injected $o b / o b$ animals. By 5 weeks, nonfasting blood glucose in AAV-hIR-injected $o b / o b$ mice dropped to a level not different from that of WT mice (Figure 4A). Similar to the blood glucose level changes observed in the ad lib-fed condition, AAV-hIR injection also caused significant decrease of fasting blood glucose (Figure 4B) and circulating plasma insulin (Figure 4C) in $o b / o b$ mice. Interestingly, compared with the dramatic glucose reduction in $o b / o b$ mice, injection of the same dose of AAV-hIR in WT mice did not result in any obvious change in glucose or insulin levels (Figure 4, A-C), which is similar to the observation in vehicle-treated iIR-KO nondiabetic mice (Figure 2A). Insulin tolerance test showed that compared with the corresponding control virus-injected groups, AAV-hIR significantly improved insulin sensitivity in $o b / o b$ mice (Figure $4, \mathrm{D}$ and F) but not in WT mice (Figure 4, E and G). Increase in liver TGs, plasma TGs, and FFAs are the hallmarks of defective lipid metabolism in $o b / o b$ mice (17). These parameters were significantly reduced (e.g., liver TGs) or completely normalized (e.g., plasma TGs and FFAs) 
A

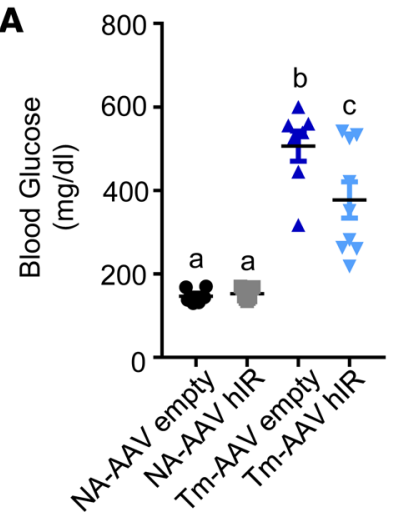

D

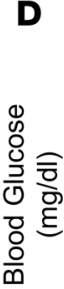

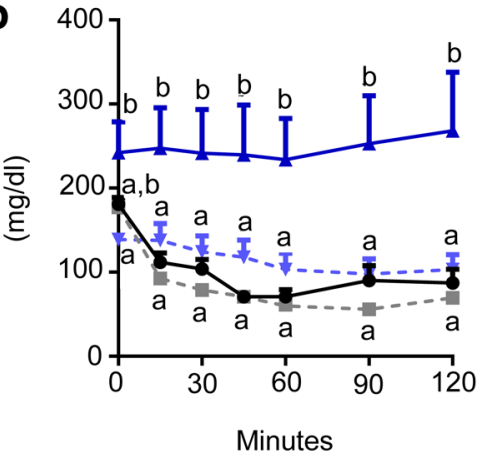

$\rightarrow$ NA-AAV empty

- NA-AAV hIR

- Tm-AAV empty

-F. Tm-AAV hIR

G

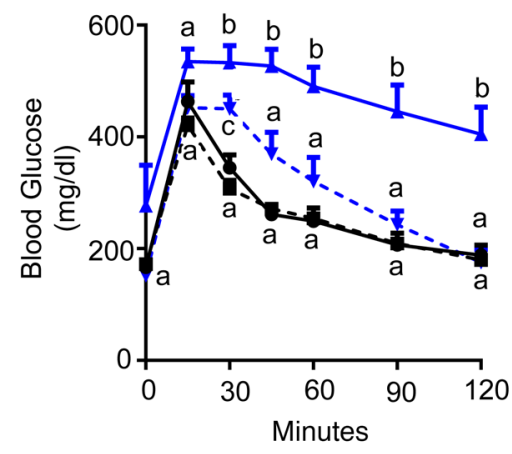

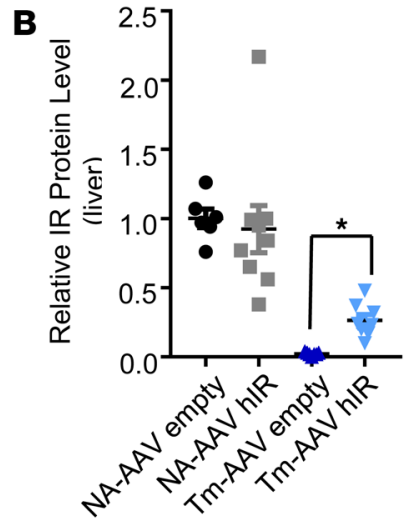

E

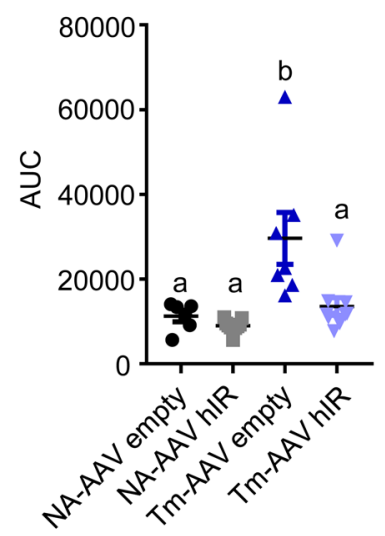

H

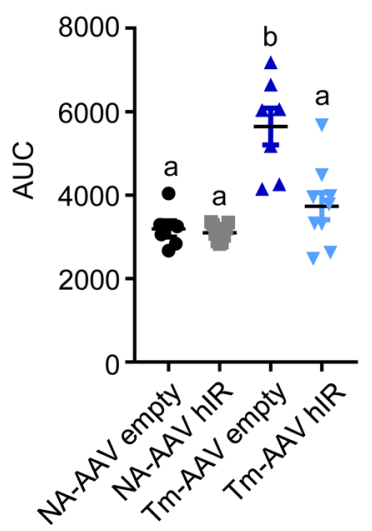

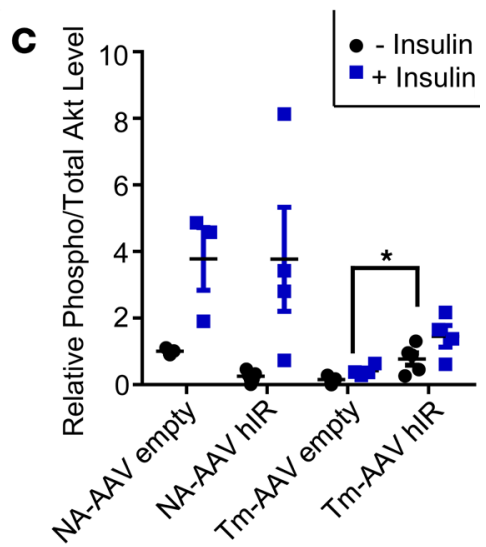

$\mathbf{F}$

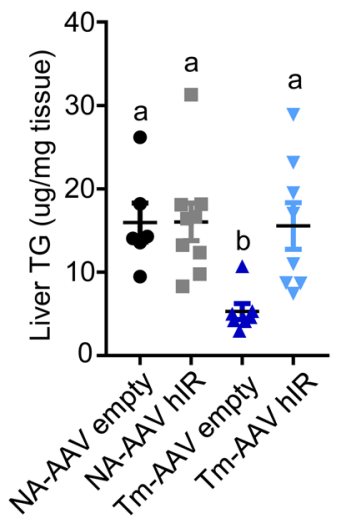

I

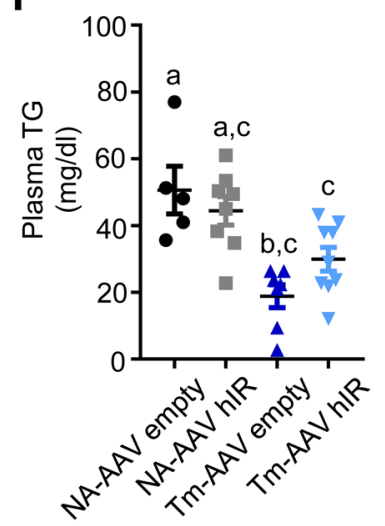

Figure 2. Injection of AAV-hIR rescued diabetic phenotype in Tm-treated iIR-KO mice. (A) Blood glucose levels were measured 3 months after AAV-hIR or control virus injection at ad lib-fed condition. (B) IR protein level $(n=6-9)$ and (C) insulin-induced Akt phosphorylation (ratio of phosphorylated to total Akt, $n=3-5$ ) in the liver were analyzed by Western blot. GAPDH was applied as an endogenous control. (D) Insulin and (C) glucose tolerance test ( $n=6-9)$. Clucose AUCs of $\mathbf{E}$ and $\mathbf{H}$ were calculated according to data in (D) and (G), respectively. Liver tissues were also subjected to (F) TC extraction and quantification. Blood was collected and subjected to (I) plasma TC measurement. Data shown as mean \pm SEM. Statistical analysis was conducted by 1-way ANOVA followed by Tukey's multiple-comparisons test $\left({ }^{*} P<0.05\right)$. Columns/data points labeled with different letters are significantly different from each other $(P<0.05)$.

by AAV-hIR injection in $o b / o b$ mice, while no change in WT mice was detected (Figure 4, H-J). These results demonstrated that AAV-hIR injection reversed glucose and lipid abnormalities in $o b / o b$ mice without causing undesirable effects, such as hypoglycemia, as highlighted in WT mice receiving AAV-hIR.

To assess AAV-hIR-induced IR expression, we analyzed IR protein levels in insulin-sensitive tissues, including liver, skeletal muscle, and eWAT. Injection of AAV-hIR rescued the diminished IR protein in livers of $o b / o b$ mice to a level comparable to that of the WT mice (Figure 5A). The normalization of IR protein lev- 
A

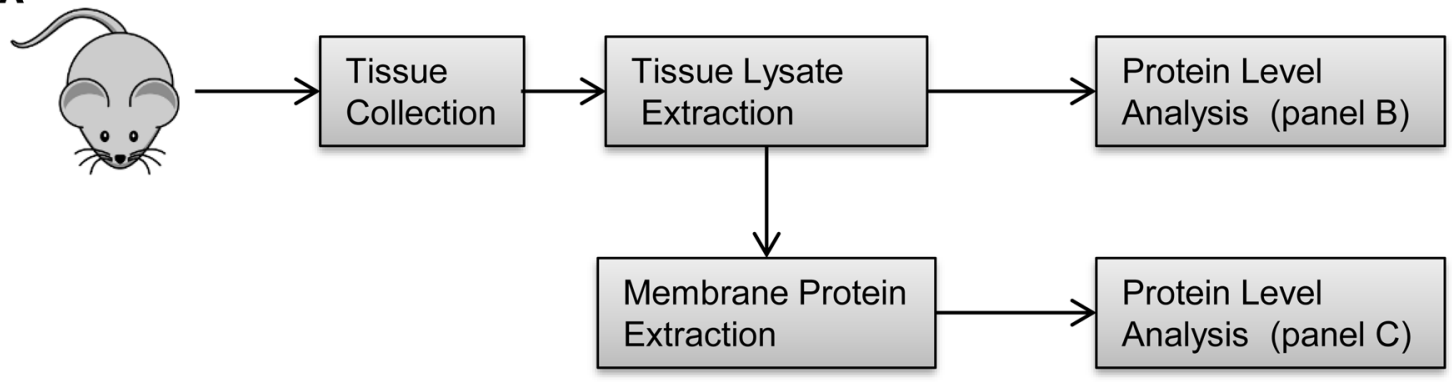

$\mathbf{B}$

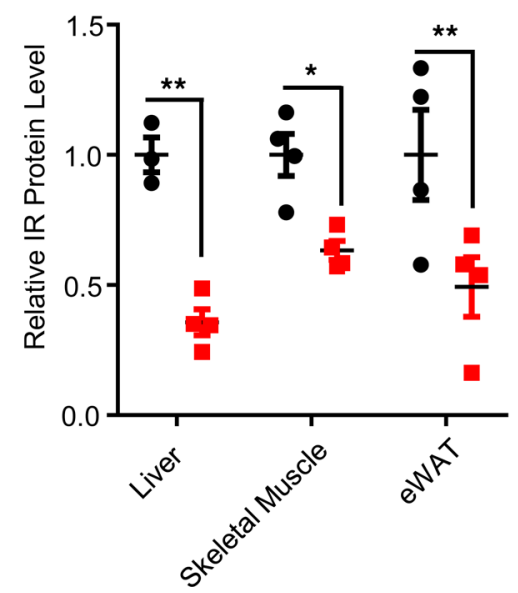

C

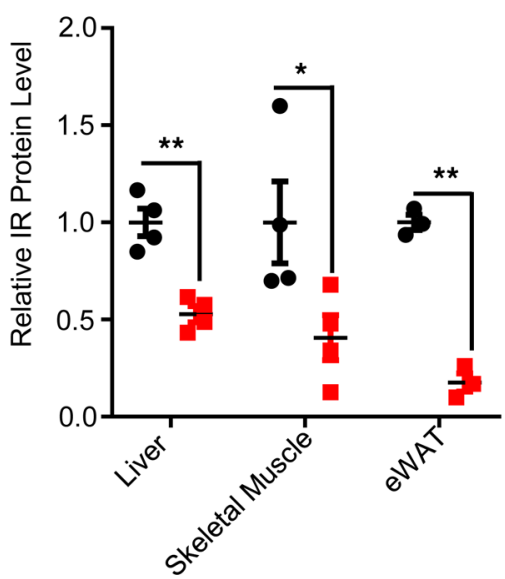

Figure 3. ob/ob mice have reduced IR protein levels in key metabolic tissues. Liver, skeletal muscle, and eWAT were collected from WT lean and $o b / o b$ mice at 4 months of age and subjected to whole-tissue lysate extraction. Membrane-bound fraction was extracted from whole-tissue lysate (A). IR levels were analyzed by Western blot using samples from (B) whole-tissue lysate or (C) membrane-bound fraction, respectively. GAPDH served as an endogenous control for each tissue $(n=3-4)$. Data presented as mean \pm SEM. Statistical analysis was conducted by $t$ test $\left({ }^{*} P<0.05 ;{ }^{* *} P<0.01\right)$.

el improved downstream insulin signaling, as indicated by the enhancement of insulin-stimulated Akt phosphorylation (Figure 5C). Corresponding to improved Akt phosphorylation, the expression of sterol regulatory element-binding protein 1 SREBP1 (a key gene that regulates hepatic de novo lipogenesis [DNL]) was significantly elevated (Supplemental Figure 6). As a comparison, significant IR protein increase was not observed in skeletal muscle (Supplemental Figure 5A) or eWAT (Supplemental Figure 5B) in $o b / o b$ mice, although there was a trend of increase in eWAT. In line with the WT mouse results described above (e.g., lack of glycemic or insulin level changes), injection of AAV-hIR in these mice did not lead to significant change of IR protein levels (Figure 5A and Supplemental Figure 5) in the insulin-sensitive tissues or insulin-stimulated hepatic Akt phosphorylation (Figure 5B). Collectively, these results demonstrated that AAV-hIR induced liver-tropic IR expression and enhanced hepatic insulin signaling and systematic insulin sensitivity in $o b / o b$ mice.

\section{Discussion}

The insulin signaling pathway participates in critical physiological functions, including cell proliferation, differentiation, growth, and metabolism (7). IR is the first step in this signaling cascade. Its important role in development is supported by the observation that whole-body deletion of IR gives rise to early postnatal death from diabetic ketoacidosis (18). The scientific debate has been ongoing regarding the primary site of defect leading to insulin resistance, i.e., the proximal versus the distal mediators in the insulin signaling cascade. It has been well documented that signaling events, such as phosphorylation of IR, IRS, PI3K, and Akt, as well as GLUT4-mediated glucose uptake, all play crucial roles in the regulation of energy metabolism, yet the primary node of insulin resistance (thus the effective targeting strategy) has not been mapped out (19). 

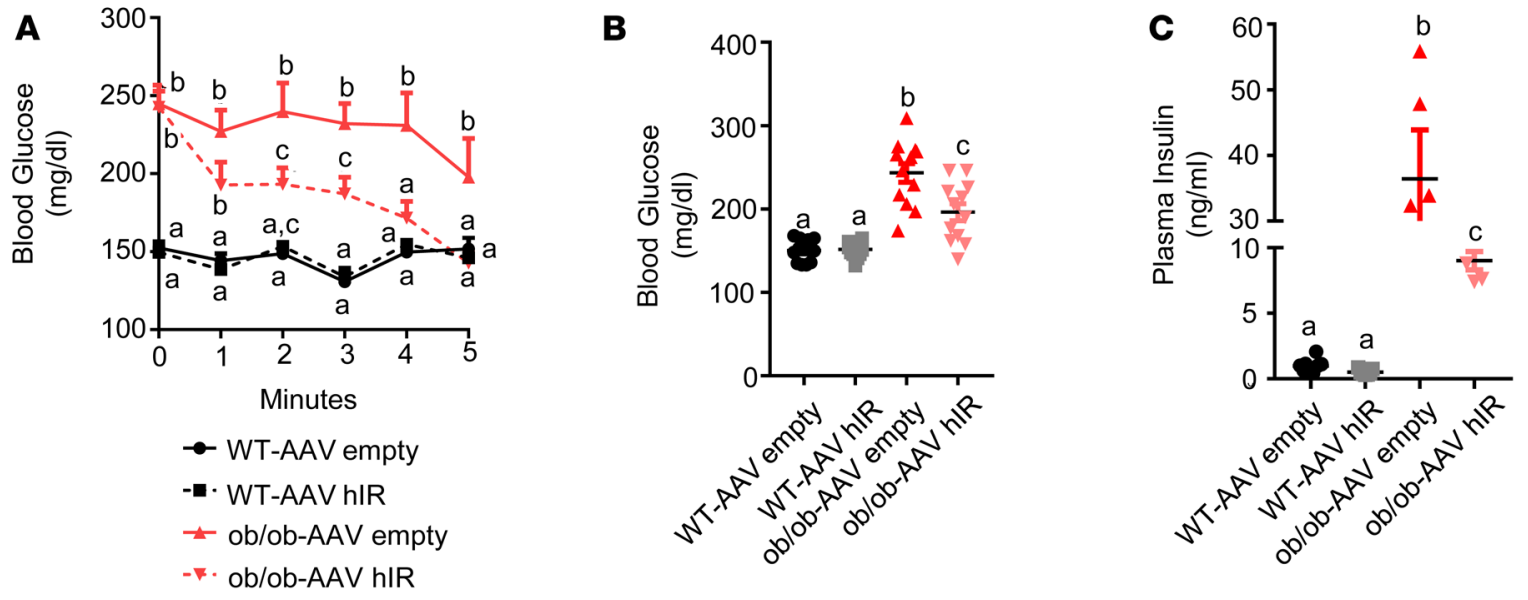
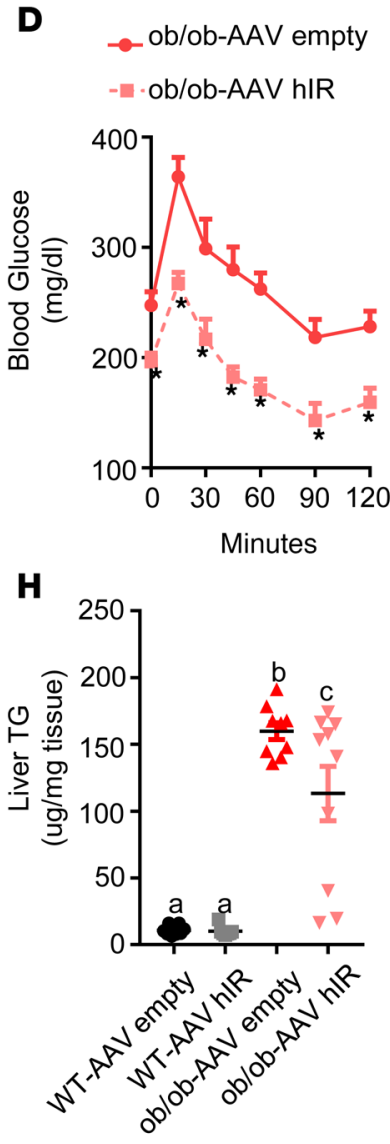

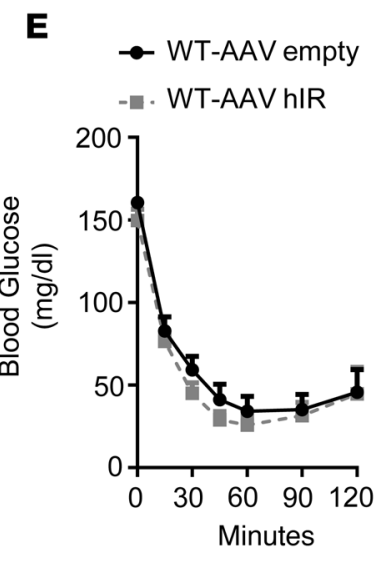

I

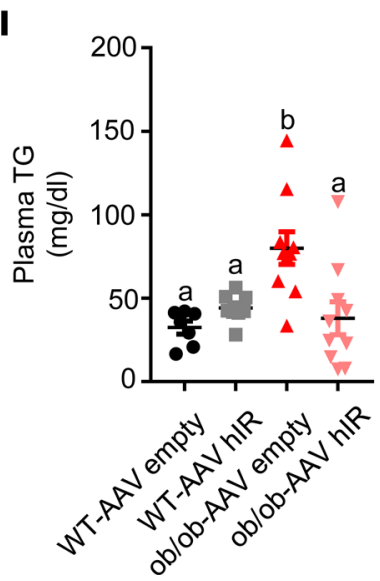

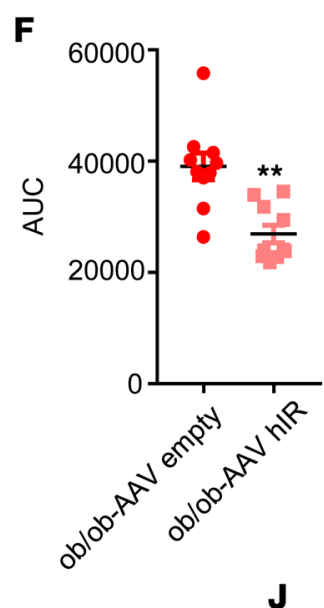
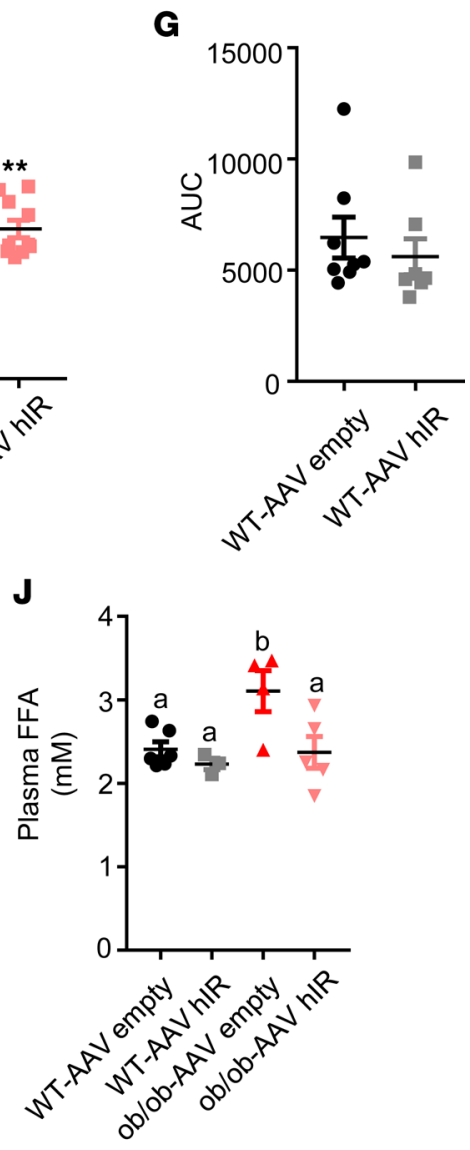

Figure 4. Injection of AAV-hIR rescued diabetic phenotype in mice. (A) Blood glucose levels were monitored from 0 to 5 weeks after AAV-hIR or control AAV injection ( $n=7-12)$. Four weeks after virus injection, WT and ob/ob mice were fasted for 5 hours and then subjected to measurements of (B) fasting blood glucose levels $(n=12)$ and (C) plasma insulin levels $(n=5-8)$. Five weeks after virus injection, (D) ob/ob and (E) WT mice were subjected to insulin tolerance test $(n=10)$. Glucose AUCs (F and $\mathbf{G})$ were calculated according to data in $\mathbf{D}$ and $\mathbf{E}$, respectively. (H) Liver TGs $(n=7-10)$, (I) plasma TGs $(n=7-10)$, and $(\mathbf{J})$ plasma FFAs $(n=3-4)$ were measured using terminal liver or blood samples. Data shown as mean \pm SEM. Statistical analysis for 2-group comparison was conducted by $t$ test $\left({ }^{*} P<0.05,{ }^{* *} P<0.01\right)$. Otherwise in the case of multiple groups, comparisons were conducted by 1-way ANOVA test followed by Tukey's multiple-comparisons test. Columns and data points marked with different letters in individual panels are significantly different from each other $(P<0.05)$.

Although deletion of FoxO1 in the liver rescued systematic glucose and insulin sensitivity in the absence of the hepatic IR, the normal state of glucose metabolism requires intact function of IR and insulin signaling from other tissues (20), which indicates the importance of IR in the whole-body metabolism. Supporting the vital role of IR in this process, aberrant IR content and kinase activity have been reported in human subjects with obesity and diabetes $(9,10,21,22)$, which may be attributed to chronic hyperinsulinemia-induced IR 
A

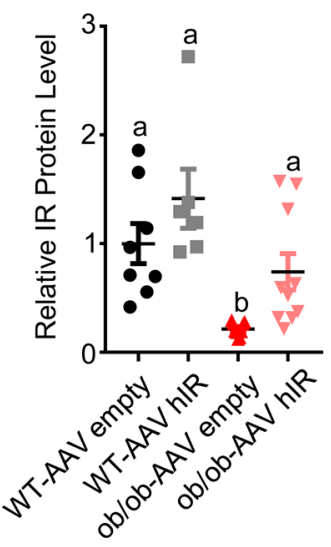

B

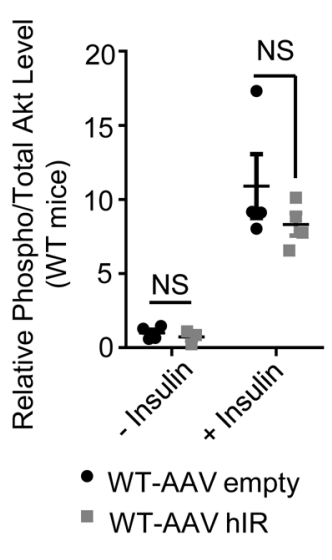

C

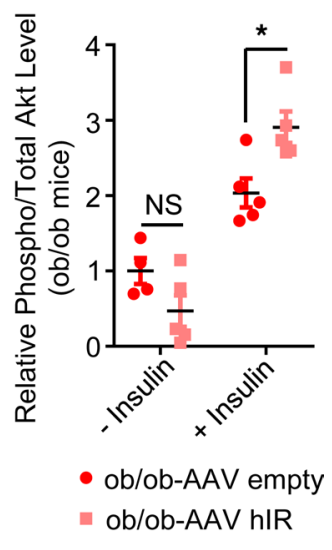

Figure 5. Injection of AAV-hIR increased IR protein and insulin-stimulated Akt phosphorylation in livers of ob/ ob mice. WT and ob/ob mice were sacrificed 5 weeks after AAV-hIR or control AAV injection. (A) Liver tissues were collected and whole-tissue lysates were subjected to Western blot for protein level analysis. GAPDH was applied as an endogenous control ( $n=6-10)$. Five weeks after virus injection, (B) WT and (C) ob/ob mice were fasted for 5 hours and then subjected to i.p. insulin injection. The ratio of phosphorylated Akt to total Akt was calculated according to Western blot results. Data shown as mean \pm SEM. Statistical analysis was conducted by 1-way ANOVA test followed by Tukey's multiple-comparisons test $(\mathbf{A})$ or $t$ test (B and $\mathbf{C},{ }^{*} P<0.05$ ). Columns labeled with different letters are significantly different from each other in the same panel $(P<0.01)$.

downregulation (23-25). Concordant with this notion, we showed that in $o b / o b$ mice, which are a model of severe obesity-associated T2DM, both total IR content and its membrane-bound fractions were decreased in these diabetic animals compared with their age-matched, lean controls. Similar observations have been made in other T2DM animals with insulin resistance. This suggests that downregulation of IR protein level may be a common mechanism contributing to the dysregulated glucose and lipid metabolism in these animals. Enhancing IR expression in different tissues via transgenic approaches has been attempted previously, the results of which were supportive of potential corrective value of restoring IR expression. However, such an approach has not been considered as a therapeutic method because in many of those studies animals bearing IR transgene did not achieve normal IR expression and were only partially rescued from diabetes, in addition to having potentially higher developmental risks $(11,13,14,26,27)$. Thus, these studies did not provide definitive conclusions on the therapeutic value of restoring IR in adult-onset diabetes as a potential cure.

In the present study, AAV9 and a truncated chicken $\beta$-actin promoter with CMV enhancer were used to deliver hIR to achieve an adequate efficiency of IR expression. AAVs drive long-lasting protein expression and cause low levels of immune response (15). AAV9 surpasses other serotypes of AAVs because of its broad genome distribution and high level of expression (28). CB promoter has been shown to deliver potent gene expression in a ubiquitous fashion (29). Restricted by the large size of the IR sequence and the limited AAV packaging capacity, a short CB promoter without the intron was applied.

The iIR-KO mouse, a model of insulin resistance due to IR deletion in multiple insulin-sensitive tissues in adult mice, was tested in the present study. This proof-of-principle experiment of IR restoration in the diabetic iIR-KO mouse by AAV delivery provided evidence about the effectiveness, tissue specificity, and safety of the current strategy. To confirm the observation in a diabetic model not driven by IR deletion as the primary defect, we applied the same strategy in $o b / o b$ mice. The successful rescue in these mice by AAV-hIR provides strong support of the current approach to treat type 2 diabetics.

Although IR is widely expressed, results from the tissue-specific KO mouse study showed that loss of IR in the liver led to severer systematic phenotype of diabetes (30) compared with IR deficiency in other tissues (31-37). This is in agreement with data supporting the liver as the primary organ of insulin action responsible for glucose and lipid metabolism $(38,39)$. In the present study, we detected a significant increase of IR protein levels upon AAV-IR treatment in the livers of $o b / o b$ and IIR-KO mice (but not in skeletal muscle or eWAT), which was sufficient to improve most of the diabetic phenotypes in these animals. The liver-tropic IR expression may be attributed to the modified CB promoter applied in the current study, which limited viral expression in tissues other than liver. In line with these findings, restoration of IR in the liver, but not in other tissues, by a transgenic approach achieved the most robust effects in rescuing 
the diabetic phenotype and extending the life span of iIR-KO mice $(11-13,27)$. These results support a prevailing role of hepatic IR over that of other tissues in the pathology of T2DM and highlight the importance of restoring IR-mediated liver metabolism via gene therapy.

In the liver, insulin suppresses hepatic glucose production and promotes lipid synthesis (40). There has been a concern that enhancing liver insulin signaling may exacerbate the hypertriglyceridemia observed in animal models and patients with T2DM. Corresponding to the enhanced Akt phosphorylation, AAV-hIR led to increased SREBP1 expression, which indicates upregulated hepatic DNL. However, hepatic IR restoration in $o b / o b$ mice reconstructed euglycemic control without hypertriglyceridemia (Figure 4, H and I). This is possibly due to reduced influx of circulating FFAs. The liver adapts 3 major lipid sources: esterified fatty acid from TG hydrolysis, nonesterified fatty acid by adipocyte lipolysis, and DNL synthesized by hepatocytes (41). As the main source of liver TGs, circulating FFAs primarily come from adipose lipolysis (42). Although AAVhIR induced liver-tropic IR expression, it improved both hepatic and systematic insulin sensitivity, which should alleviate the insulin resistance in eWAT and slow down the FFA influx into the liver as indicated by the normalization of circulating FFA levels (Figure 4J). Similar to what was observed in $o b / o b$ mice, we detected FFA change in the same direction, albeit milder, upon AAV-hIR treatment in iIR-KO mice (Supplemental Figure 3). These data demonstrated the correlation between glycemia improvement and adipocyte lipolysis inhibition. The decrease of ectopic lipid accumulation mitigates inflammation in liver and forms a positive feedback loop to improve insulin sensitivity (43). A recent study provided evidence that increased liver lipid accumulation may be attributable to insulin resistance of the peripheral tissues (44) because hepatic TG synthesis is more dependent on increased plasma fatty acid esterification than hepatic insulin signaling (45).

Insulin promotes hepatic TG synthesis and inhibits lipolysis in WAT. In WAT of $o b / o b$ mice, elevation of FFAs indicates unopposed lipolysis due to insulin resistance (46). Although the current strategy restored insulin signaling in $o b / o b$ mice, it did not increase hepatic TG level beyond the level of the control animals. This suggests, in the current models, the inhibitory role of lipolysis in WAT prevails over hepatic TG synthesis with regard to hepatic TG regulation by insulin signaling.

Interestingly, although applied to both healthy and T2DM animals, the current approach did not increase IR protein beyond its physiological level in the healthy animals (Figure 2B and Figure 5A). Because insulin signaling is involved in multiple vital cellular functions, the protein level of IR needs to be tightly controlled. One of the negative regulations is protein degradation by adaptor protein and E3 ligases to circumvent excessive activation (47). Upregulation of the E3 ligases has been proposed as a mechanism of IR protein reduction under insulin-resistant status (48-50). It is possible that AAV-mediated IR delivery is expressed and maintained only as "needed," and the negative regulation mechanisms keep IR protein within its physiological range, which is critical to protect animals from deadly hypoglycemia and the risk of tumorigenesis as a consequence of IR pathway overactivation. A similar observation has been reported in a study of L1 mice, a strain of IR-KO mice bearing an IR transgene (13). Although L1 mice have IR mRNA level much higher than that of WT or other knockin lines, IR protein level in L1 mice was not the highest among the transgenic lines, and none of them exceeded the level of IR seen in WT mice (13). Collectively, these data support the idea that endogenous checkpoints of IR protein level help prevent excessive IR expression and subsequent overstimulation of IR pathway. Further investigation of the molecular mechanisms will shed light on the precise pathway protecting cells from excessive insulin action.

In the present study, we demonstrated that a single treatment with AAV-hIR delivery can induce liver-tropic IR expression and rescue the majority of the diabetic phenotypes in the T2DM models. As a proof of principle, these results indicated that a similar approach can be applied to adult-onset T2DM as a potential gene therapy to control glycemia and correct other metabolic abnormalities safely and effectively.

\section{Methods}

Animals and in vivo studies. Animals were maintained under controlled conditions of 12-hour light/12-hour dark cycles, temperature $\left(23^{\circ} \mathrm{C} \pm 2^{\circ} \mathrm{C}\right)$, and humidity $(55 \% \pm 15 \%)$ with chow diet ad lib provided unless specified. All the animals applied in the current study were male. WT and $o b / o b$ mice and the donor mice for generating IIR-KO mice were obtained from Jackson Laboratory. We selected $o b / o b$ mice from those with blood glucose of 200 to $300 \mathrm{mg} / \mathrm{dL}$. DIO mice were fed with high-fat diet (60\% of total energy from fat) for 10 weeks. All diets were from Research Diets, Inc. The iIR-KO mice were generated and bred at Taconic Farm.

Tm treatment in the current study was executed by 5 continuous i.p. injections $(30 \mathrm{mg} / \mathrm{kg}$ body weight, 1 injection/day). The solution of Tm was freshly prepared for each injection. One night before injection, 
Tm was dissolved in corn oil at $10 \mathrm{mg} / \mathrm{mL}$ and incubated at $37^{\circ} \mathrm{C}$ overnight, and the injection was performed the next morning. The animals of control groups were injected with corn oil vehicle of the same volume. For experiments of chronic effect observation, Tm treatment was repeated every month, which is the approximately effective period of Tm for inducing Cre-loxP recombination $(51,52)$.

For testing insulin or glucose tolerance, animals were fasted for 5 hours before being subjected to i.p. insulin injection (iIR-KO mice: $1.5 \mathrm{U} / \mathrm{kg}$ body weight; WT mice: $0.5 \mathrm{U} / \mathrm{kg}$ body weight; $o b / o b$ mice: $4 \mathrm{U} /$ $\mathrm{kg}$ body weight) or i.p. glucose injection at $2 \mathrm{~g} / \mathrm{kg}$ body weight (iIR-KO mice). Blood glucose levels were measured at the indicated time points shown in the figures. For testing insulin-induced Akt phosphorylation, animals were fasted for 5 hours before being subjected to i.p insulin injection (iIR-KO mice: $1.5 \mathrm{U} / \mathrm{kg}$ body weight; WT mice: $0.5 \mathrm{U} / \mathrm{kg}$ body weight; $o b / o b$ mice: $4 \mathrm{U} / \mathrm{kg}$ body weight). Animals were sacrificed 30 minutes after insulin injection. After animal sacrifice, tissue samples were snap-frozen in liquid nitrogen immediately and stored in $-80^{\circ} \mathrm{C}$ till further analysis.

Blood glucose levels were measured by OneTouch Ultra meter (LifeScan). Plasma insulin was measured by ELISA (Mercodia). Hepatic TGs were extracted using methods previously described (53, 54). Plasma and hepatic TG concentrations were measured using Triglycerides Liquid Stable Reagent (Thermo Fisher Scientific). Plasma FFAs were measured using Free Fatty Acids, Halt Micro Test (Roche).

Production of AAV9-hIR and control AAVs. The IR gene (NM_000208.3) was synthesized at Genewiz Inc. The synthesized gene was subcloned into AflII and SpeI sites of a backbone pAAV cis plasmid that contained a truncated chicken $\beta$-actin promoter with CMV enhancer (short CB promoter) to accommodate the size of IR gene, and the resulting pAAV cis plasmid was termed AAV-hIR (Supplemental Figure 1). AAV9-hIR and the negative control AAV vector that contained the short $\mathrm{CB}$ promoter were produced by the helper-free triple-plasmid transfection method as described previously (55). The AAV cis plasmid, an adenoviral helper plasmid and a packaging plasmid containing the AAV2 Rep gene and AAV9 Cap gene were cotransfected into HEK293 cells. AAV vectors were subsequently purified by 2 rounds of cesium chloride density gradient ultracentrifugation. The titers of AAV vectors were determined via reverse transcription PCR analysis, and the purity of the AAV preps was determined by silver staining. The produced viruses were stored at $-80^{\circ} \mathrm{C}$. Both AAV9-hIR and control (AAV9 empty) vector were freshly diluted at $5 \times 10^{12}$ genome copies (GC)/mL in phosphate-buffered saline and then administered to animals at $100 \mu \mathrm{L} /$ mouse dosage via tail vein injection.

Antibodies and reagents. Anti-GAPDH monoclonal antibody was purchased from MBL International Corporation (catalog M171-3). Antibodies against GAPDH (catalog 2118), IR (catalog 3025), total AKT (catalog 4691), and phospho-AKT Ser473 (catalog 4051) were purchased from Cell Signaling Technology. All Western blot reagents were purchased from ProteinSimple. Protease inhibitor (Halt Protease Inhibitor Cocktail) was purchased from Thermo Fisher Scientific. Tm, corn oil, and phosphatase inhibitor cocktails 2 and 3 were purchased from MilliporeSigma.

Western blotting. Tissue samples were homogenized in $4^{\circ} \mathrm{C}$ using radioimmunoprecipitation assay buffer with protease inhibitor and phosphatase inhibitors. After incubation at $4^{\circ} \mathrm{C}$ for 30 minutes with constant rotating, the homogenized tissue samples were centrifuged at $18,000 \mathrm{~g}$ and $4^{\circ} \mathrm{C}$ for 10 minutes, and supernatants were isolated as whole-tissue lysate samples. Protein concentration was determined using bicinchoninic acid assay (Thermo Fisher Scientific). Membrane-bound fraction was further extracted using Mem-PER Plus Membrane Protein Extraction Kit (Thermo Fisher Scientific). Protein lysates were denatured at $37^{\circ} \mathrm{C}$ for 60 minutes with dithiothreitol provided in the standard pack 1 from ProteinSimple and then analyzed using the Simple Western size-based capillary electrophoresis system (ProteinSimple).

$R N A$ extraction and real-time quantitative PCR. Total RNA from frozen tissues was extracted by using SV Total RNA Isolation System (Promega). cDNA was generated by reverse transcription using SuperScript VILO cDNA synthesis kit (Invitrogen), which was then assigned as a template for quantitative PCR. Gene expression of SREBP1 (Mm00550338_m1) and GAPDH (Mm99999915_g1) was amplified by using TaqMan gene expression assays with the $\Delta \Delta \mathrm{Ct}$ method for quantification (Thermo Fisher Scientific). GAP$\mathrm{DH}$ in this experiment served as the endogenous internal control.

Statistics. Statistical analysis was performed using GraphPad Prism 7.02 software. All data were presented as mean \pm SEM. Two-tailed $t$ test was applied to 2-group data analysis, and 1-way ANOVA followed by Tukey's multiple-comparisons test was applied to multigroup data analysis. A $P$ value less than 0.05 was considered significant.

Study approval. All animal procedures in the present study were reviewed and approved by the Institutional Animal Care and Use Committee of Merck and Co., Inc., Kenilworth, New Jersey, USA. 


\section{Author contributions}

YW and JM designed the research. HZ designed AAV vectors applied in the research. YW and OP performed the research. YW analyzed the data. YW and JM wrote the manuscript.

\section{Acknowledgments}

We would like to thank Liming Yang, Margaret Wu, Ge Dai, Xiaolan Shen, Christian Nunes, and Xiaoli Ping for technical support and Jing Li for critical review of the manuscript. The research is solely sponsored by Merck and Co., Inc., Kenilworth, New Jersey, USA.

Address correspondence to: James Mu, 630 Gateway Blvd., South San Francisco, California 94080, USA. Phone: 650.496.4507; Email: yingjun_mu@merck.com.

1. Saltiel AR, Kahn CR. Insulin signalling and the regulation of glucose and lipid metabolism. Nature. 2001;414(6865):799-806.

2. Reach G, Pechtner V, Gentilella R, Corcos A, Ceriello A. Clinical inertia and its impact on treatment intensification in people with type 2 diabetes mellitus. Diabetes Metab. 2017;43(6):501-511.

3. Kitabchi AE, Umpierrez GE, Miles JM, Fisher JN. Hyperglycemic crises in adult patients with diabetes. Diabetes Care. 2009;32(7):1335-1343.

4. Mathers CD, Loncar D. Projections of global mortality and burden of disease from 2002 to 2030. PLoS Med. 2006;3(11):e442.

5. Chen L, Magliano DJ, Zimmet PZ. The worldwide epidemiology of type 2 diabetes mellitus — present and future perspectives. Nat Rev Endocrinol. 2011;8(4):228-236.

6. Kadowaki T, Ueki K, Yamauchi T, Kubota N. SnapShot: insulin signaling pathways. Cell. 2012;148(3):624, 624.e1.

7. Boucher J, Kleinridders A, Kahn CR. Insulin receptor signaling in normal and insulin-resistant states. Cold Spring Harb Perspect Biol. 2014;6(1):a009191.

8. Tiwari S, Halagappa VK, Riazi S, Hu X, Ecelbarger CA. Reduced expression of insulin receptors in the kidneys of insulin-resistant rats. J Am Soc Nephrol. 2007;18(10):2661-2671.

9. Ojamaa K, et al. Defects in human insulin receptor gene expression. Mol Endocrinol. 1988;2(3):242-247.

10. Chiefari E, et al. Functional variants of the HMGA1 gene and type 2 diabetes mellitus. JAMA. 2011;305(9):903-912.

11. Baudry A, et al. Partial rescue of insulin receptor-deficient mice by transgenic complementation with an activated insulin receptor in the liver. Gene. 2002;299(1-2):219-225.

12. Lin HV, Accili D. Reconstitution of insulin action in muscle, white adipose tissue, and brain of insulin receptor knock-out mice fails to rescue diabetes. J Biol Chem. 2011;286(11):9797-9804.

13. Okamoto H, Nakae J, Kitamura T, Park BC, Dragatsis I, Accili D. Transgenic rescue of insulin receptor-deficient mice. J Clin Invest. 2004;114(2):214-223.

14. Sasaki T, et al. Overexpression of insulin receptor partially improves obese and diabetic phenotypes in $\mathrm{db} / \mathrm{db}$ mice. Endocr $J$. 2015;62(9):787-796.

15. Naso MF, Tomkowicz B, Perry WL, Strohl WR. Adeno-associated virus (AAV) as a vector for gene therapy. BioDrugs. 2017;31(4):317-334.

16. Harris VM. Protein detection by Simple Western analysis. Methods Mol Biol. 2015;1312:465-468.

17. Picard F, Richard D, Huang Q, Deshaies Y. Effects of leptin adipose tissue lipoprotein lipase in the obese ob/ob mouse. Int J Obes Relat Metab Disord. 1998;22(11):1088-1095.

18. Accili D, et al. Early neonatal death in mice homozygous for a null allele of the insulin receptor gene. Nat Genet. 1996;12(1):106-109

19. Hoehn KL, et al. IRS1-independent defects define major nodes of insulin resistance. Cell Metab. 2008;7(5):421-433.

20. O-Sullivan I, et al. FoxO1 integrates direct and indirect effects of insulin on hepatic glucose production and glucose utilization. Nat Commun. 2015;6:7079.

21. Arner P, Pollare T, Lithell H, Livingston JN. Defective insulin receptor tyrosine kinase in human skeletal muscle in obesity and type 2 (non-insulin-dependent) diabetes mellitus. Diabetologia. 1987;30(6):437-440.

22. Caro JF, et al. Insulin receptor kinase in human skeletal muscle from obese subjects with and without noninsulin dependent diabetes. J Clin Invest. 1987;79(5):1330-1337.

23. Kasuga M, Kahn CR, Hedo JA, Van Obberghen E, Yamada KM. Insulin-induced receptor loss in cultured human lymphocytes is due to accelerated receptor degradation. Proc Natl Acad Sci U S A. 1981;78(11):6917-6921.

24. Lay AC, et al. Prolonged exposure of mouse and human podocytes to insulin induces insulin resistance through lysosomal and proteasomal degradation of the insulin receptor. Diabetologia. 2017;60(11):2299-2311.

25. McElduff A, Hedo JA, Taylor SI, Roth J, Gorden P. Insulin receptor degradation is accelerated in cultured lymphocytes from patients with genetic syndromes of extreme insulin resistance. J Clin Invest. 1984;74(4):1366-1374.

26. Lauro D, et al. Impaired glucose tolerance in mice with a targeted impairment of insulin action in muscle and adipose tissue. Nat Genet. 1998;20(3):294-298.

27. Okamoto H, Obici S, Accili D, Rossetti L. Restoration of liver insulin signaling in Insr knockout mice fails to normalize hepatic insulin action. J Clin Invest. 2005;115(5):1314-1322.

28. Zincarelli C, Soltys S, Rengo G, Rabinowitz JE. Analysis of AAV serotypes 1-9 mediated gene expression and tropism in mice after systemic injection. Mol Ther. 2008;16(6):1073-1080.

29. Powell SK, Rivera-Soto R, Gray SJ. Viral expression cassette elements to enhance transgene target specificity and expression in gene therapy. Discov Med. 2015;19(102):49-57.

30. Michael MD, et al. Loss of insulin signaling in hepatocytes leads to severe insulin resistance and progressive hepatic dysfunc- 
tion. Mol Cell. 2000;6(1):87-97.

31. Brüning JC, et al. A muscle-specific insulin receptor knockout exhibits features of the metabolic syndrome of NIDDM without altering glucose tolerance. Mol Cell. 1998;2(5):559-569.

32. Brüning JC, et al. Role of brain insulin receptor in control of body weight and reproduction. Science. 2000;289(5487):2122-2125.

33. Guerra C, et al. Brown adipose tissue-specific insulin receptor knockout shows diabetic phenotype without insulin resistance. $J$ Clin Invest. 2001;108(8):1205-1213.

34. Blüher M, et al. Adipose tissue selective insulin receptor knockout protects against obesity and obesity-related glucose intolerance. Dev Cell. 2002;3(1):25-38.

35. Otani K, et al. Reduced $\beta$-cell mass and altered glucose sensing impair insulin-secretory function in betaIRKO mice. Am J Physiol Endocrinol Metab. 2004;286(1):E41-E49.

36. Zhang J, Liu F. Tissue-specific insulin signaling in the regulation of metabolism and aging. IUBMB Life. 2014;66(7):485-495.

37. Konishi M, et al. Endothelial insulin receptors differentially control insulin signaling kinetics in peripheral tissues and brain of mice. Proc Natl Acad Sci U S A. 2017;114(40):E8478-E8487.

38. Tokarz VL, MacDonald PE, Klip A. The cell biology of systemic insulin function. J Cell Biol. 2018;217(7):2273-2289.

39. Bechmann LP, Hannivoort RA, Gerken G, Hotamisligil GS, Trauner M, Canbay A. The interaction of hepatic lipid and glucose metabolism in liver diseases. J Hepatol. 2012;56(4):952-964.

40. Sanders FW, Griffin JL. De novo lipogenesis in the liver in health and disease: more than just a shunting yard for glucose. Biol Rev Camb Philos Soc. 2016;91(2):452-468.

41. Donnelly KL, Smith CI, Schwarzenberg SJ, Jessurun J, Boldt MD, Parks EJ. Sources of fatty acids stored in liver and secreted via lipoproteins in patients with nonalcoholic fatty liver disease. J Clin Invest. 2005;115(5):1343-1351.

42. Paschos P, Paletas K. Non alcoholic fatty liver disease and metabolic syndrome. Hippokratia. 2009;13(1):9-19.

43. Samuel VT, Shulman GI. Mechanisms for insulin resistance: common threads and missing links. Cell. 2012;148(5):852-871.

44. Vatner DF, et al. Evidence against pathway-selective hepatic insulin resistance in mice. Moderated poster discussion at: American Diabetes Association's 78th Scientific Sessions; June 22-26, 2018; Orlando, Florida, USA.

45. Vatner DF, et al. Insulin-independent regulation of hepatic triglyceride synthesis by fatty acids. Proc Natl Acad Sci U S A. 2015;112(4):1143-1148.

46. Song Z, Xiaoli AM, Yang F. Regulation and metabolic significance of de novo lipogenesis in adipose tissues. Nutrients. 2018;10(10):E1383.

47. Tawo R, et al. The ubiquitin ligase CHIP integrates proteostasis and aging by regulation of insulin receptor turnover. Cell. 2017;169(3):470-482.e13.

48. Song R, et al. Central role of E3 ubiquitin ligase MG53 in insulin resistance and metabolic disorders. Nature. 2013;494(7437):375-379.

49. Nagarajan A, et al. MARCH1 regulates insulin sensitivity by controlling cell surface insulin receptor levels. Nat Commun. 2016;7:12639.

50. Ramos FJ, Langlais PR, Hu D, Dong LQ, Liu F. Grb10 mediates insulin-stimulated degradation of the insulin receptor: a mechanism of negative regulation. Am J Physiol Endocrinol Metab. 2006;290(6):E1262-E1266.

51. Reinert RB, et al. Tamoxifen-induced Cre-loxP recombination is prolonged in pancreatic islets of adult mice. PLoS One. 2012;7(3):e33529.

52. Sakaguchi M, et al. Adipocyte dynamics and reversible metabolic syndrome in mice with an inducible adipocyte-specific deletion of the insulin receptor. Cell Metab. 2017;25(2):448-62.

53. Bligh EG, Dyer WJ. A rapid method of total lipid extraction and purification. Can J Biochem Physiol. 1959;37(8):911-917.

54. $\mathrm{Mu}$ J, et al. Chronic inhibition of dipeptidyl peptidase- 4 with a sitagliptin analog preserves pancreatic $\beta$-cell mass and function in a rodent model of type 2 diabetes. Diabetes. 2006;55(6):1695-1704.

55. Chen Z, et al. AAV8-mediated long-term expression of human LCAT significantly improves lipid profiles in hCETP;Ldlr(+/-) mice. J Cardiovasc Transl Res. 2011;4(6):801-810. 